\title{
Clinicopathological Characteristics of Microscopic Tubal Intraepithelial Metastases from Adenocarcinoma and Small Cell Neuroendocrine Carcinoma of the Uterine Cervix
}

\author{
JIYEON LEE ${ }^{1}$, SUJIN PARK ${ }^{1}$, HA YOUNG WOO ${ }^{2,3}$ and HYUN-SOO KIM ${ }^{1}$ \\ ${ }^{1}$ Department of Pathology and Translational Genomics, Samsung Medical Center, \\ Sungkyunkwan University School of Medicine, Seoul, Republic of Korea; \\ ${ }^{2}$ Department of Pathology, National Cancer Center, Goyang-si, Republic of Korea; \\ ${ }^{3}$ Department of Pathology, Severance Hospital, Yonsei University College of Medicine, Seoul, Republic of Korea
}

\begin{abstract}
Background/Aim: Some metastatic tumors that involve the fallopian tube show intraepithelial spread, mimicking primary tubal neoplasm and representing a potential diagnostic pitfall. In this study, we aimed to investigate the clinicopathological characteristics of tubal intraepithelial metastasis (IEM) from cervical carcinoma. Patients and Methods: We analyzed the clinical features, histological features, and immunophenotypes of IEMs in five patients with cervical carcinoma. Results: This study included usual-type (1/5), mucinous-type (1/5), and gastrictype (2/5) endocervical adenocarcinomas and small cell neuroendocrine carcinoma (1/5) cases. None of the patients had ovarian metastasis, but metastatic tumor cells spread along the tubal mucosal surface and partially replaced the lining epithelium. Histological features of metastatic tumors closely resembled those of the primary tumors in all cases. Conclusion: Tubal IEM can mimic various tubal lesions including serous tubal intraepithelial carcinoma. Morphological consistency between the primary and metastatic tumors and immunostaining help guide the differential diagnosis of challenging intraepithelial lesions of the fallopian tube.
\end{abstract}

This article is freely accessible online.

Correspondence to: Hyun-Soo Kim, Department of Pathology and Translational Genomics, Samsung Medical Center, Sungkyunkwan University School of Medicine, 81, Irwon-ro, Gangnam-gu, Seoul 06351, Republic of Korea. Tel: +82 234101243, Fax: +82 234142831, e-mail: hyun-soo.kim@ samsung.com; Ha Young Woo, Department of Pathology, National Cancer Center, 323, Ilsan-ro, Ilsandong-gu, Goyang-si, Gyeonggi-do 10408, Republic of Korea. Tel: +82 319201739, Fax: +82 319201369, e-mail: why@ncc.re.kr

Key Words: Fallopian tube, intraepithelial metastasis, uterus, cervix, endocervical adenocarcinoma, small cell neuroendocrine carcinoma.
Cervical carcinoma is one of the commonest causes of carcinoma-related mortality among women worldwide. The incidence and mortality rates of cervical carcinoma have decreased significantly in developed countries since the introduction of the cervicovaginal cytology screening program, which has facilitated the detection and treatment of precursor lesions (i.e. high-grade squamous intraepithelial lesion and adenocarcinoma in situ) (1). Despite the high efficacy of local treatment for the precursor lesions, treated women continue to represent a high-risk group, with high recurrence rates of 5-10\% (2). Furthermore, despite increased surveillance, for several years after treatment, these women have a higher risk of invasive carcinoma than women in the general population (3). In 2018, approximately 570,000 women developed cervical carcinoma and 311,000 women died from it $(4,5)$.

Squamous cell carcinoma is the commonest histological subtype arising in the uterine cervix, which accounts for $70 \%$ of all cervical carcinoma cases (6). Endocervical adenocarcinoma and high-grade neuroendocrine carcinoma comprise approximately $25 \%$ and $2 \%$, respectively $(6,7)$. A recent large-scale study revealed that the most frequent anatomic site of metastatic cervical carcinoma was the lungs (37.9\%), followed by the bone (16.7\%), liver (12.5\%), and brain $(1.6 \%)(8)$. Adnexal metastasis of cervical carcinoma is relatively uncommon. The incidence of ovarian metastases ranges $2.0-28.6 \%$ and $0-17.4 \%$ for endocervical adenocarcinomas and squamous cell carcinomas, respectively. The clinicopathological characteristics of cervical carcinoma that involves the ovaries have been well documented (9), whereas only a few studies have investigated metastatic cervical carcinoma to the fallopian tube (10).

Most of the tubal metastases from gynecological malignancies are associated with ovarian metastases (10). Tubal metastasis that occurs alone is generally asymptomatic and grossly unidentifiable (11). Metastatic tumors involve 
typically one or more microanatomical locations, including the serosa, myosalpinx, endosalpinx, and lymphovascular space. However, sometimes, they ingeniously replace the lining epithelium of the normal tubal mucosa but without stromal invasion, closely resembling serous tubal intraepithelial carcinoma (STIC) (12). These lesions are evident only upon microscopic examination (12). The intraepithelial growth of metastatic tumor cells along the surface epithelium can be overlooked or misdiagnosed as STIC (11). In this study, we describe a series of five cases wherein endocervical adenocarcinomas and small cell neuroendocrine carcinoma (SCNEC) metastasized to the unilateral or bilateral fallopian tubes. In particular, this case series comprises cervical carcinomas that had metastasized only to the surface epithelium without invasion of the subepithelial stroma. We conducted this study with the aim to comprehensively investigate the clinicopathological characteristics of tubal intraepithelial metastasis (IEM) in association with cervical carcinoma.

\section{Patients and Methods}

Case selection. The study protocol was reviewed and approved (2020-10-134) by the Institutional Review Board of the Samsung Medical Center (Seoul, Republic of Korea). We searched the surgical pathology database of the Severance Hospital (Seoul, Republic of Korea) and the Samsung Medical Center (Seoul, Republic of Korea) and included two and three cases, respectively, of cervical carcinoma with tubal microscopic IEM. We collected clinical information, including the patient's age; previous gynecological history, presenting symptoms, magnetic resonance imaging (MRI) findings; serum levels of cancer antigen (CA) 125, CA 19-9, and carcinoembryonic antigen (CEA); preoperative clinical impression; type of surgical procedure; postoperative recurrence and distant metastasis; disease-free survival; current status, and overall survival, from the electronic medical records or from the referring physicians.

Pathological examination. Two board-certified gynecological pathologists thoroughly examined all of the available hematoxylin and eosin-stained slides by light microscopy. We collected pathological information, including the cervical punch biopsy result; histological subtype and microscopically measured greatest dimension of primary cervical tumor; extension into the uterine corpus (endomyometrium), upper vagina, and parametrium; lymphovascular space invasion; intraoperative peritoneal fluid cytology; metastasis to the peritoneum, lymph node, and distant organ at the time of surgery; initial pathological International Federation of Gynecology and Obstetrics (FIGO) stage; the presence of grossly visible adnexal mass; ovarian and tubal metastasis; and the greatest dimension, extent, multifocality, and distribution of tubal metastatic tumor. For each case, the most representative slide was selected for immunostaining.

Immunostaining. Briefly, 4- $\mu$ m-thick, formalin-fixed, paraffinembedded slices were deparaffinized and rehydrated using a xylene and alcohol solution. Immunostaining was performed using the
Bond Polymer Intense Detection System (Vision Biosystems, Mount Waverly, Victoria, Australia) (13-19). After antigen retrieval, the slices were incubated with primary antibodies including estrogen receptor (ER; 1:150, clone 6F11, Novocastra, Leica Biosystems, Newcastle Upon Tyne, UK), progesterone receptor (PR; 1:100, clone 16, Novocastra), p53 (1:300, clone DO-7, Novocastra), p16 (prediluted, clone E6H4, Ventana Medical Systems, Oro Valley, AZ, USA), neural cell adhesion molecule (CD56, 1:200, clone CD564, Novocastra), chromogranin A (1:800, clone DAK-A3, Dako, Agilent Technologies, Inc., Santa Clara, CA, USA), synaptophysin (1:400, clone DAKO-SYNAP, Dako), and Wilms tumor 1 (WT1, 1:800, clone 6F-H2, Cell Marque, Rocklin, CA, USA). After chromogenic visualization, the slices were counterstained with hematoxylin. Appropriate positive and negative controls were concurrently stained to validate the staining method. Endometrial endometrioid (for ER and PR) and serous (for p16 and p53) carcinomas were used as positive controls. Ovarian high-grade serous carcinoma (for WT1) and pulmonary SCNEC (for CD56, chromogranin A, and synaptophysin) were also used as positive controls. Negative controls were prepared by substituting non-immune serum for primary antibodies, which resulted in no detectable staining.

The staining intensity (weak, moderate, or strong) and proportion (focal or diffuse) were evaluated in the nucleus (for ER, PR, and WT1), cytoplasm (for chromogranin and synaptophysin), and cell membrane (for CD56). The p53 immunostaining pattern was interpreted as a mutant pattern when one of the following staining patterns was observed: diffuse and strong nuclear immunoreactivity in $75 \%$ or more of the tumor cells (over-expression pattern); no nuclear immunoreactivity in any of the tumor cells (complete absence pattern); and an unequivocal diffuse cytoplasmic staining (cytoplasmic pattern) (20). In contrast, p53 expression was interpreted as a wild-type pattern if a variable proportion of tumor cell nuclei expressed p53 protein with mild-to-moderate staining intensity (14, $21,22)$. The p16 immunostaining pattern was interpreted as block positive when p16 expression was horizontally continuous and strong, and involved nuclear or nuclear plus cytoplasmic staining. All other p16 immunostaining patterns-described as focal nuclear staining or wispy, blob-like, puddled, or scattered cytoplasmic staining-were interpreted as patchy positive $(13,14,21-27)$.

\section{Results}

Clinical features. Table I summarizes the clinical features of all five patients included in this series. Their age ranged from 42 to 64 years (mean=54.4 years). None of the patients had any gynecological history. All except one patient presented with vaginal discharge. Imaging findings were available for all patients. On MRI, the mean greatest dimension of cervical tumors was $4.2 \mathrm{~cm}$ (range=2.6-7.1 cm). Two patients showed suspected and definite upper vaginal extension, respectively, whereas the vagina appeared to be tumor-free in the remaining three patients. MRI revealed no definite parametrial extension in all except one patient. Similarly, definite pelvic lymph node metastases were observed in one patient. Serum levels of CA 125, CA 19-9, and CEA were examined in five, four, and two patients, respectively. The serum CA 125 level was within the normal range in all patients, whereas serum levels of CA 19-9 (117.8 U/ml) and 


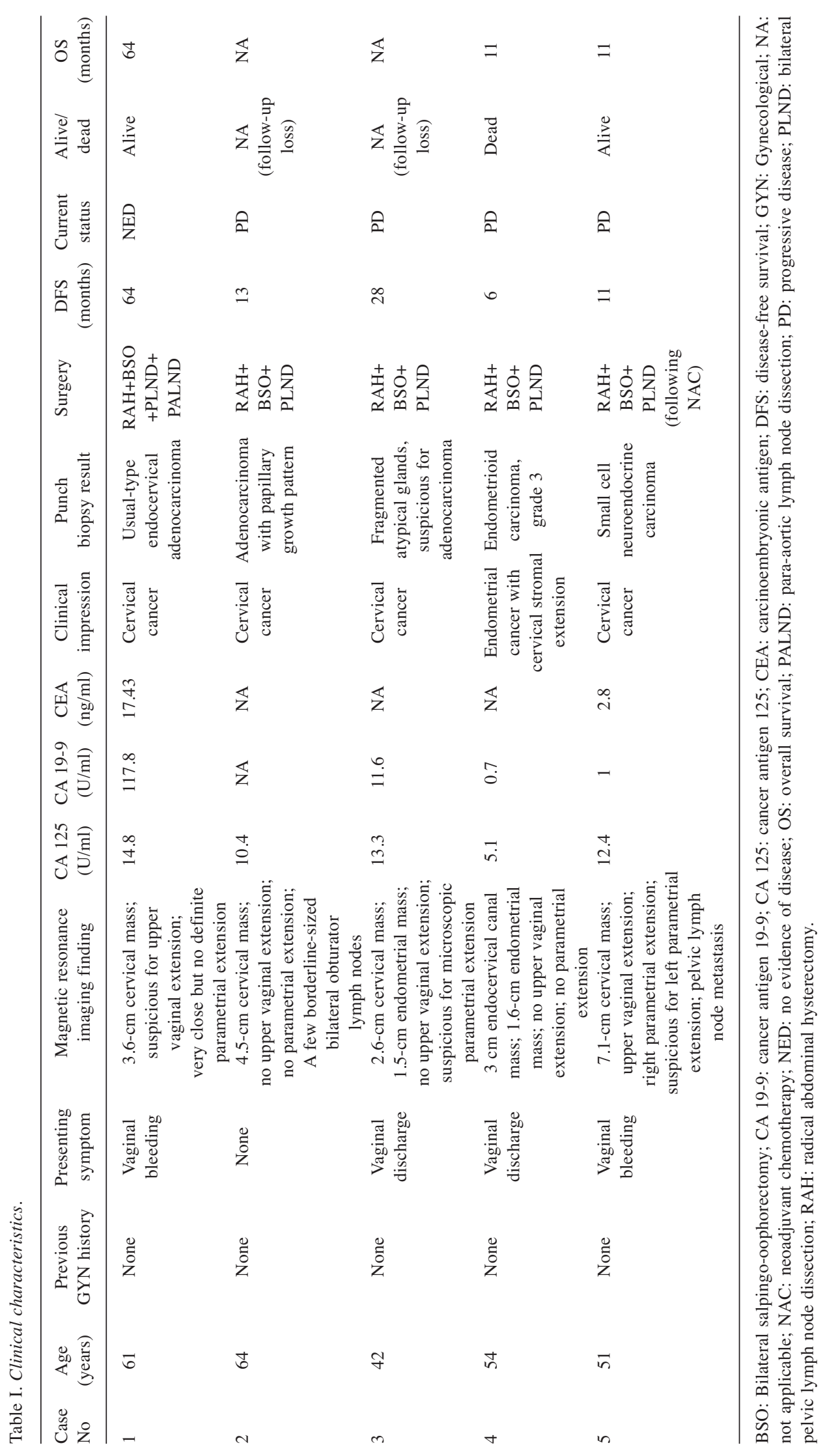


CEA $(17.43 \mathrm{ng} / \mathrm{ml})$ were elevated in one patient (case 1). Four of the five patients were diagnosed preoperatively with cervical carcinoma. In the remaining patient, endometrial carcinoma with cervical stromal extension was suspected because the imaging findings showed two masses in the endocervical canal and endometrium; thus, the biopsy was interpreted as indicating grade 3 endometrioid carcinoma of the endometrium. All patients underwent surgery as the primary treatment, including radical abdominal hysterectomy $(5 / 5)$, bilateral salpingo-oophorectomy $(5 / 5)$, bilateral pelvic lymph node dissection (5/5), and para-aortic lymph node dissection (1/5). One patient (case 5) received preoperative neoadjuvant chemotherapy. All patients underwent postoperative concurrent chemoradiation therapy.

Four patients (cases 2-5) developed distant metastases in the liver, lungs, omentum, peritoneum, or pancreas, with a mean disease-free survival time of 14.5 months (range $=6-28$ months). The survival data of two patients (cases 2 and 3) who were lost to follow-up after chemotherapy were unavailable. Among the three patients whose follow-up data were available, one (case 4) died of metastatic disease at 11 months postoperatively, and another (case 5) is alive with multi-organ distant metastases at 11 months postoperatively. The remaining patient (case 1) is alive without disease recurrence or metastasis at 64 months postoperatively.

Pathological features. Table II summarizes the pathological features. Two patients were diagnosed with HPV-associated endocervical adenocarcinoma (usual and mucinous type, respectively). HPV-independent endocervical adenocarcinoma of gastric type was diagnosed in another two patients. The remaining patient was diagnosed with SCNEC. The microscopically measured mean greatest dimension of cervical tumors was $41 \mathrm{~mm}$ (range $=14-65$ $\mathrm{mm}$ ). Four tumors extensively involved the uterine corpus (extensive endomyometrial involvement), and all tumors infiltrated the parametrium. Three patients had upper vaginal tumor extension. Intraoperative peritoneal washing cytology was evaluated in one patient (case 4) and was positive for malignant cells, but peritoneal metastasis was not confirmed histologically in the resected specimen. All patients had pelvic lymph node metastases. One patient (case 5) had distant metastasis at the time of surgery, whereas three patients (cases 2-4) postoperatively developed distant metastases. Taken together, initial pathological FIGO stages were IIB (2/5), IIIC (2/5), and IVB (1/5).

Pathological features of tubal metastatic tumors. Table III summarizes the pathological features of tubal IEMs. None of the patients had a grossly identifiable mass in the adnexa. All ovaries showed no pathological abnormality. All tubes had multifocal microscopic foci of metastatic carcinoma that were confined within the surface epithelium of the bilateral tubes $(2 / 5)$ or unilateral tube (3/5), with the greatest dimension measuring no more than $2 \mathrm{~mm}$. In all cases, metastatic tumors involved not only the distal portions, including the fimbria and infundibulum, but also the ampulla and isthmus. A detailed description of the histological features of the five cases is presented here.

Case 1 (HPV-associated endocervical adenocarcinoma of usual type). Metastatic usual-type endocervical adenocarcinoma displayed a mucin-poor, pseudostratified columnar epithelium possessing enlarged, hyperchromatic, elongated nuclei. The neoplastic epithelium spread along the surface epithelial lining and partially replaced it (Figure 1A), with abrupt transitions from apparently normal cuboidal-tolow columnar epithelium to darkly stained, irregularly thickened epithelium (Figure 1B). On high-power magnification, the tumor cells exhibited severe nuclear enlargement and pleomorphism compared to the adjacent normal tubal epithelium (Figure 1C). The amount of cytoplasm was moderate, but the intracytoplasmic mucin was not readily identifiable. Frequent apical mitoses and apoptotic bodies, both of which are characteristics of HPV-associated endocervical adenocarcinoma, were observed. Several microscopic metastatic lesions $(\leq 2 \mathrm{~mm}$ ) were randomly distributed in both the fimbrial and non-fimbrial portions, although they did not invade the subepithelial stroma. Immunostaining revealed that all the neoplastic epithelium displayed block p16 positivity (Figure 1D), but contrasting tested negative for WT1 (Figure 1E), ER (Figure 1F), and PR, as well as wild-type p53 immunostaining pattern.

Case 2 (HPV-associated endocervical adenocarcinoma of mucinous type). We noted the foci of complex glandular proliferation with intraluminal papillary projections, which partially replaced the tubal epithelium (Figure 1G). Immunohistochemically, the neoplastic epithelium was negative for WT1, which reacted intensely with nonneoplastic tubal epithelium (Figure 1H). The dilated tubal lumen contained an admixture of eosinophilic mucinous material, inflammatory cells, and cellular debris. The tumor cells possessed abundant endocervical-type intracytoplasmic mucin. Occasional goblet cells were noted. Furthermore, in the transverse sections of the ampulla, we observed that the normal tubal epithelium was nearly replaced completely by the metastatic carcinoma (Figure 1I). Immunostaining revealed that p16 intensely highlighted tumor cells that extended horizontally along the epithelium (Figure 1J). There was no stromal invasion. Architectural patterns and nuclear features were similar to those of the usual-type endocervical adenocarcinoma observed in case 1 , but the presence of intracytoplasmic mucin in the majority of tumor cells supported a diagnosis of metastatic mucinous-type endocervical adenocarcinoma. 


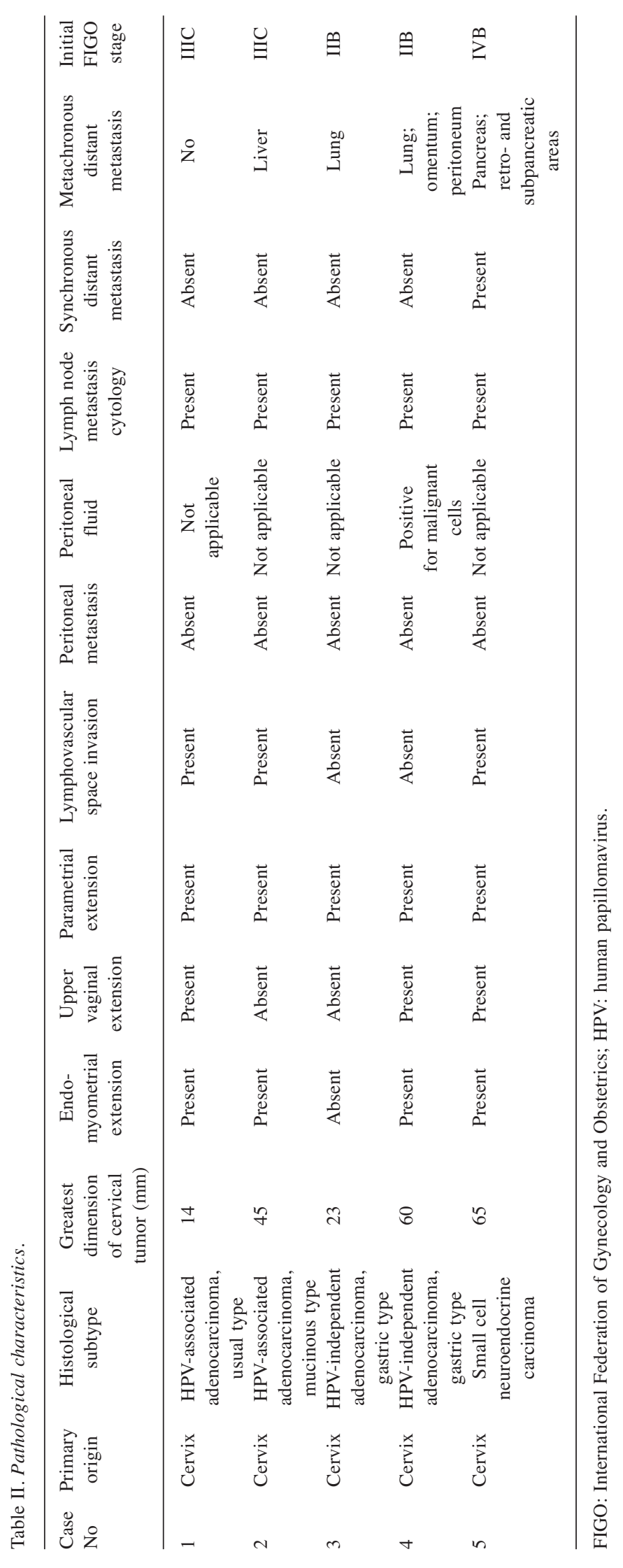


Table III. Pathological characteristics of tubal metastatic tumors.

\begin{tabular}{lccccccc}
\hline $\begin{array}{c}\text { Case } \\
\text { No }\end{array}$ & $\begin{array}{c}\text { Grossly visible } \\
\text { adnexal mass }\end{array}$ & $\begin{array}{c}\text { Ovarian } \\
\text { metastasis }\end{array}$ & $\begin{array}{c}\text { Tubal } \\
\text { metastasis }\end{array}$ & $\begin{array}{c}\text { Greatest dimension of } \\
\text { tubal metastatic tumor (mm) }\end{array}$ & $\begin{array}{c}\text { Extent of tubal } \\
\text { metastatic tumor }\end{array}$ & Multifocality & Distribution \\
\hline 1 & Absent & Absent & Present & 2 & Intraepithelial (left) & Multifocal & Fimbrial and non-fimbrial \\
2 & Absent & Absent & Present & 2 & Intraepithelial (bilateral) & Multifocal & Fimbrial and non-fimbrial \\
3 & Absent & Absent & Present & 2 & Intraepithelial (right) & Multifocal & Fimbrial and non-fimbrial \\
4 & Absent & Absent & Present & 2 & Intraepithelial (bilateral) & Multifocal & Fimbrial and non-fimbrial \\
5 & Absent & Absent & Present & 1 & Intraepithelial (right) & Multifocal & Fimbrial and non-fimbrial \\
\hline
\end{tabular}
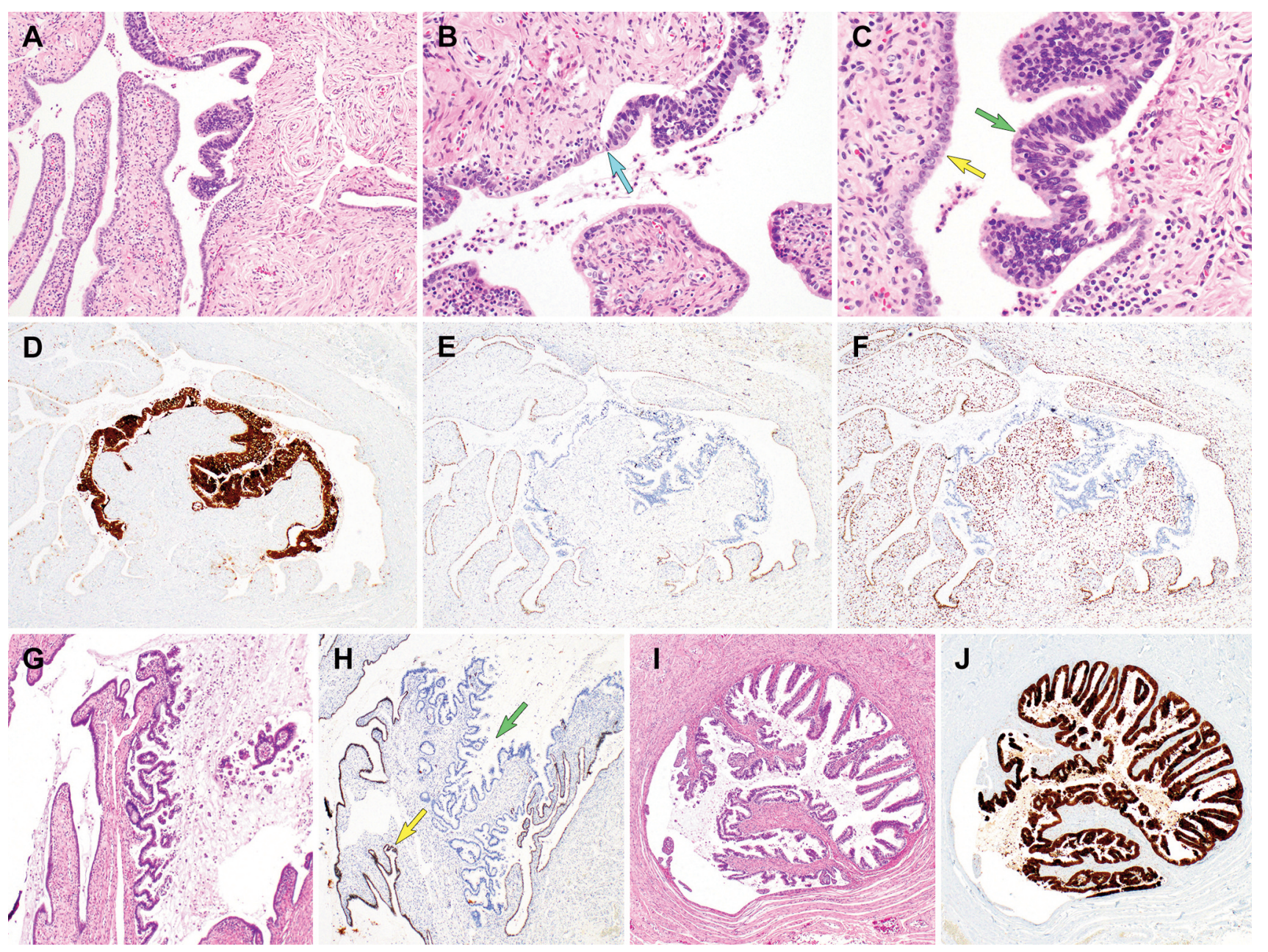

Figure 1. Histological features and immunophenotype of tubal intraepithelial metastases from usual-type (A-F; case 1) and mucinous-type (G-J; case 2) endocervical adenocarcinoma. (A) Case 1. The tubal epithelium is partially replaced by markedly thickened epithelium. No stromal invasion is noted. (B) There is an abrupt transition from apparently normal epithelium to severely atypical epithelium (blue arrow). (C) High-power magnification reveals that metastatic carcinoma (green arrow) exhibits severe pleomorphism and nuclear stratification compared to the adjacent normal tubal epithelium (yellow arrow). The amount of cytoplasm is moderate, but the intracytoplasmic mucin is not easily identifiable. Mitotic figures and apoptotic bodies are frequently observed. (D-F) Immunostaining reveals that the tumor cells display (D) block p16 positivity and lack of (E) Wilms tumor 1 and $(F)$ estrogen receptor expression. $(G)$ Case 2. Complex glandular proliferation replaces the tubal epithelium. The dilated glandular lumen contains mucin and cellular debris, supporting the diagnosis of metastatic mucinous-type endocervical adenocarcinoma. (H) The neoplastic glands (green arrow) are negative for Wilms tumor 1, which reacts intensely with normal tubal epithelium (yellow arrow). (I) One cross section of the ampulla shows the involvement of almost all of the epithelium by metastatic carcinoma. Stromal invasion is absent. (J) p16 immunostaining highlights the tumor cells that spread horizontally along and the epithelium. Original magnification, A-B, 100x; C, 200x; D-J, 40x. 
Case 3 (HPV-independent endocervical adenocarcinoma of gastric type). The metastatic tumor showed identical morphology to that of the primary cervical tumor: atypical glandular epithelium possessing enlarged, hyperchromatic nuclei and abundant, pale-to-eosinophilic cytoplasm. Similar to the distribution patterns observed in cases 1 and 2, multifocal microscopic metastases were confined within the tubal epithelium (Figure 2A). In the fimbria, infundibulum, and ampulla, the tumor cells proliferated along the mucosal surface. Abrupt transitions between normal and neoplastic epithelium were noted. Some microscopic tumor emboli were detected within the tubal lumina. The tumor cells possessed eccentrically located nuclei showing pseudostratification, enlargement, and moderate-to-severe pleomorphism. Occasional mitoses and conspicuous nucleoli were present; intracytoplasmic mucin vacuoles were often observed on the apical surface. Microscopic segmental replacement of the fimbrial serous-type epithelium with atypical cells showing nuclear hyperchromasia and pleomorphism as well as mitotic figures closely resembled STIC. Immunostaining revealed that the tumor cells were highlighted by p53 immunostaining (Figure 2B), which showed diffuse and strong nuclear expression (p53 overexpression). In contrast, tumor cells were negative for WT1 (Figure 2C) and hormone receptors (Figure 2D). In the areas of abrupt transition (Figure 2E-F), the tumor cells displayed p53 over-expression (Figure 2G) but lacked WT1 expression (Figure 2H).

Case 4 (HPV-independent endocervical adenocarcinoma of gastric type). The morphological features observed in case 4 were similar to those in case 3. Metastatic gastric-type endocervical adenocarcinoma displayed abundant intracytoplasmic mucin (Figure 2I). The basally located nuclei of tumor cells were large and pleomorphic, with conspicuous nucleoli (Figure 2J). p53 immunostaining highlighted the epithelium-limited involvement of metastatic carcinoma under the low-power view (Figure 2K). The tumor cells strongly expressed p53. The intraoperative peritoneal washing cytology specimen revealed small, tight cellular clusters (Figure 2L). On high-power magnification, the tumor cells showed hyperchromatic, pleomorphic nuclei and high nuclear-to-cytoplasmic ratio (Figure $2 \mathrm{M}$ and $\mathrm{N}$ ). Their nuclei were eccentrically located due to distension by intracytoplasmic mucin (Figure 2O). Thus, the sample was cytologically diagnosed as positive for malignant cells (i.e., metastatic carcinoma).

Case 5 (SCNEC). The primary tumor destructively infiltrated the entire cervical stroma as well as the parametrium and upper vagina (Figure 3A). The tumor mainly comprised solid, compact sheets of tumor cells, although large areas of geographic tumor cell necrosis were frequently noted (Figure
3B). The tumor cells were small, and their cytoplasm was scant and delicate (Figure 3C). The tumor cell nuclei displayed frequent molding and crush artifact, salt-andpepper chromatin pattern, marked pleomorphism, inconspicuous nucleoli, and brisk mitotic activity. We found that the right tube contained numerous, small microscopic foci of IEM (Figure 3D). The greatest dimension of each focus did not exceed $1 \mathrm{~mm}$ (Figure 3E). The mucosal involvement of metastatic carcinoma simulated the thickened tubal epithelium of chronic salpingitis. Moreover, the normal tubal epithelium was replaced by tumor cells showing nuclear hyperchromasia and pleomorphism as well as brisk mitotic activity (Figure 3F), resembling STIC. Moreover, in some foci, the individual tumor cells extended laterally along the adjacent tubal mucosa and were distributed underneath the normal epithelium, resembling pagetoid spread (Figure 3G-H). The tumor cells tested positive for CD56 (Figure 3I) but negative for WT1 (Figure 3J) on immunostaining. The tumor cell cytoplasm reacted positively for synaptophysin (Figure 3K) and chromogranin A (Figure 3L), both of which are reliable markers of neuroendocrine differentiation.

\section{Discussion}

Herein, we presented five cases of cervical carcinoma that metastasized to the fallopian tube and described their detailed clinical and pathological aspects, including histological features and immunostaining results of the tubal metastases. Despite the small number of cases, we focused on the various histological subtypes of cervical carcinoma: HPV-associated endocervical adenocarcinoma of usual and mucinous types, HPV-independent endocervical adenocarcinoma of gastric type, and SCNEC. We demonstrated that the tubal metastatic tumors were confined within the mucosa and spread along the surface. In all cases, the adnexa appeared grossly unremarkable, and there was no ovarian metastasis. Instead, the fallopian tubes exhibited microscopic IEMs, which were distributed over not only the distal but also the middle and proximal portions of the tube. Four of the five patients showed extension into the upper vagina and parametrium, which was not identified in the preoperative imaging studies.

From the pathologists' point of view, tubal metastases from cervical carcinomas should be distinguished from various tubal conditions: STIC, high-grade serous carcinoma (HGSC), and other non-neoplastic lesions, including AriasStella reaction, tubal hyperplasia, secretory cell outgrowth (SCOUT), and metaplastic changes (28). The colonization of metastatic tumor cells on the tubal mucosa can mimic primary tubal neoplasm. High-grade nuclear atypia, including loss of polarity, enlargement, hyperchromasia, moderate-to-severe pleomorphism, prominent nucleoli, apoptotic bodies, and increased mitotic activity, is a sign that 


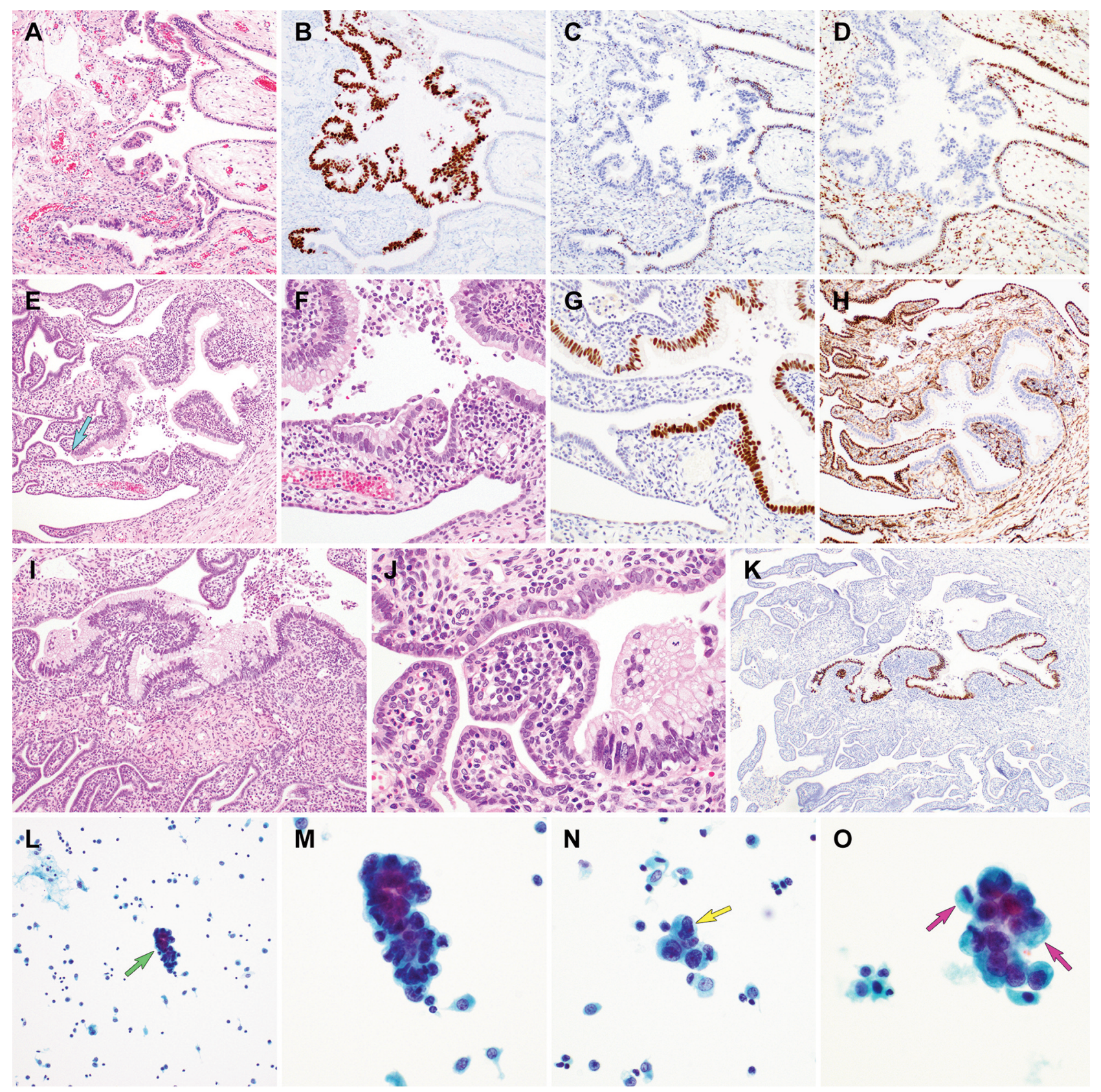

Figure 2. Histological features, immunophenotype, and cytological features of tubal intraepithelial metastases from gastric-type endocervical adenocarcinoma (cases 3 and 4). (A) Case 3. The metastatic lesion shows intraepithelial colonization of complex glandular proliferation. (B) The tumor cells are highlighted by p53 immunostaining, which reveals diffuse and strong nuclear expression. $(C$ and D) Immunostaining for $(C)$ Wilms tumor 1 and $(D)$ estrogen receptor reveals uniform nuclear expression in normal tubal epithelium but the absence of expression in the tumor cells. (E) Neoplastic glandular cells proliferate along and replace the tubal epithelium. An abrupt transition (blue arrow) between normal tubal epithelium and neoplastic glandular epithelium is noted. At low-power magnification, the neoplastic glands form dilated lumina and appear to possess larger nuclei and apical intracytoplasmic mucin. (F) High-power magnification reveals the neoplastic glandular epithelium with nuclear stratification and pleomorphism. Intracytoplasmic mucin vacuoles are present on the apical surface. ( $G$ and $H)$ Immunohistochemically, the tumor cells display $(G)$ diffuse and strong nuclear p53 immunoreactivity $(H)$ and Wilms tumor 1 negativity. (I) Case 4. The tumor cells display abundant intracytoplasmic mucin and basally located nuclei. (J) The tumor cells have large, pleomorphic nuclei with conspicuous nucleoli. (K) p53 highlights the intraepithelial location of metastatic carcinoma. The tumor distribution is easily identified by strong nuclear p53 expression. (L) Ascitic fluid cytology reveals a small, tight cellular cluster (green arrow). ( $M$ and $N$ ) At high-power magnification, the tumor cells show hyperchromatic, pleomorphic nuclei (yellow arrow) and high nuclear-to-cytoplasmic ratio. $(O)$ The nuclei are eccentrically located due to distension by intracytoplasmic mucin (purple arrows). Staining method, $A, E, F, I$, and $J$, hematoxylin and eosin staining; $B-D, G, H$, and $K-L$, polymer method; L-O, Papanicolaou staining. Original magnification, A-E, 100x; F-G, 200x; H-I, 100x; J, 400x; K-L, 40x; M-O, 400x. 


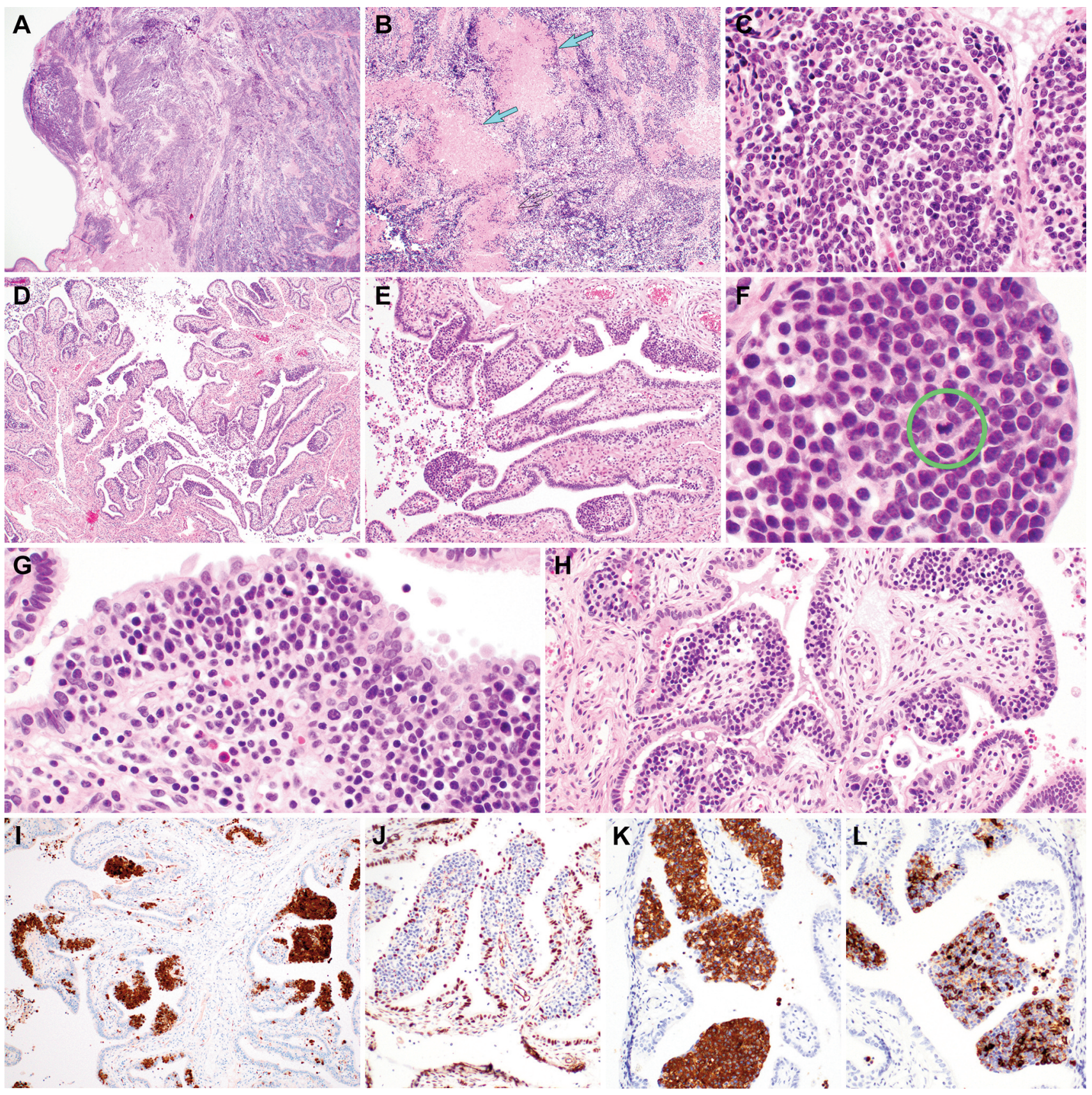

Figure 3. Histological features and immunophenotype of tubal intraepithelial metastases from small cell neuroendocrine carcinoma. (A) A large tumor mass involving the uterine cervix shows hypercellular sheets of tumor cells. It infiltrates extensively the cervical stroma. (B) The tumor exhibits multiple foci of coagulative tumor cell necrosis (blue arrows). (C) The tumor cells are small-sized cells with less than the diameter of three small resting lymphocytes. They show indistinct cell border, scant cytoplasm, nuclear hyperchromasia and molding, irregular nuclear membrane, high nuclear-to-cytoplasmic ratio. (D) There are multifocal microscopic foci of metastatic carcinoma along the tubal surface. (E) The mucosal involvement of metastatic carcinoma simulates thickened tubal epithelium, chronic salpingitis, or serous tubal intraepithelial carcinoma. (F) Highpower magnification reveals tumor cells possessing scant cytoplasm and hyperchromatic nuclei with salt-and-pepper chromatin, molding, irregular membrane, inconspicuous nucleoli, and mitotic figures (green circle). These histological features of tubal metastatic tumor are identical to those of the primary cervical tumor. ( $G$ and $H$ ) The tumor cells infiltrate the tubal epithelium. At $(G)$ medium-and $(H)$ low-power magnifications, the nuclear size of tumor cells is similar to or slightly larger than that of lymphocytes and plasma cells. The individual tumor cells extend laterally along the adjacent tubal mucosa and are distributed underneath the normal epithelium, resembling pagetoid spread. (I-L) Immunohistochemically, the tumor cells are positive for (I) CD56 but negative for (J) Wilms tumor 1. (K) Synaptophysin and $(L)$ chromogranin A, both of which are markers of neuroendocrine differentiation, present within the tumor cell cytoplasm. Staining method, A-H, hematoxylin and eosin staining; I-L, polymer method. Original magnification, A, 40x; B, 100x; C, 400x; D, 40x; E, 100x; F, 600x; G, 400x; H, 200x, I, 100x; and J-L, 100x. 
is suggestive of malignancy and may be a characteristic of both metastatic tumor and STIC (28). In other words, the degree of architectural or cytological atypia cannot be used as the key criteria for differentiating metastatic lesions from STIC. Papillary tufting and slit-like glandular spaces indicate the possibility of STIC but are inconclusive. There are some differences in histological features between IEM and STIC. Firstly, unlike metastatic lesions, the majority of STIC arises from the fimbria (29). Secondly, as the metastatic tumor cells closely resemble the primary tumor, morphological consistency between the primary and metastatic tumors can help to differentiate between tubal metastasis and STIC. Usual-type endocervical adenocarcinoma (case 1) shows numerous apically situated mitotic figures and basally situated apoptotic debris appreciable at scanning magnification $(28,30)$. Tumors with mucinous components, including mucinous-type (case 2) and gastric-type (cases 3 and 4) endocervical adenocarcinoma, show intracytoplasmic mucin and may have some goblet cells, both of which are not seen in STIC. The tumor cell nuclei of SCNEC (case 5) display frequent molding, salt-and-pepper chromatin pattern, marked pleomorphism, inconspicuous nucleoli, and brisk mitotic activity (31). HGSCs present typically as grossly evident mass-forming lesions with ovarian involvement and tubal stromal invasion. The Arias-Stella reaction can occur in the tubal epithelium of pregnant women, generating a similar morphology to that seen in the endometrium. This benign condition can be distinguished from malignant lesions based on clinical information about a recent pregnancy. Tubal hyperplasia primarily occurs in patients with estrogen-producing tumors or in response to inflammatory diseases such as pelvic inflammatory disease and endometriosis (32). Histologically, tubal hyperplasia shows several features, including a cribriform, back-to-back tubular epithelium, or microcystic sieve-like growth pattern, that induce suspicion of a neoplastic condition (33). However, there is an absence of both high-grade nuclear atypia and atypical mitotic figures. SCOUT is a rare condition, which may look distinct from the adjacent tubal epithelium. SCOUT lacks block p16 positivity and highgrade cytological atypia and shows wild-type p53 immunostaining pattern.

The metaplastic change of the tubal epithelium is a benign, reactive cytoplasmic alteration. The commonest type is mucinous metaplasia, whereas transitional and squamous metaplasias are less common. In patients with malignant tumors showing mucinous differentiation, it is often difficult for pathologists to determine whether the tubal lesion is a metastatic lesion or mucinous metaplasia. Few studies have investigated mucinous metaplasia of the fallopian tube. Wong et al. (34) reported 23 cases of tubal mucinous metaplasia, and only one of them was identified in a patient with colonic carcinoma. The authors suggested that, as mucinous cytoplasmic alterations of the tubal epithelium are observed in patients who do not have a malignant tumor, these changes represent a metaplastic process. Furthermore, they stated that the incidence of tubal mucinous metaplasia remains unknown, and is probably underreported. Moreover, there are no reliable immunohistochemical markers for tubal mucinous metaplasia because its immunophenotype was seldom investigated. Unlike metastatic mucinous carcinoma, mucinous metaplasia lacks cytological atypia and mitotic activity, and tends to coexist with other metaplastic changes and chronic inflammation. Nevertheless, the possibility of neoplastic transformation of tubal mucinous metaplasia should be considered when atypical mucinous glandular proliferation is associated with cytological and architectural atypia.

Immunostaining can guide the differential diagnosis of challenging intraepithelial lesions of the fallopian tube. WT1 is the first marker to be included in the immunostaining panel. STIC shows uniform positivity for WT1, whereas WT1 expression was absent in all five cases of metastatic cervical carcinoma. A mutant p53 immunostaining pattern is another requisite feature for diagnosing STIC. Most endocervical adenocarcinomas, particularly HPV-associated tumors, demonstrate wild-type p53 expression pattern, which helps to distinguish metastatic lesions from STIC. However, one potential pitfall that is worthy of mention is that either p53 over-expression or complete absence of p53 immunoreactivity is observed in a significant proportion of gastric-type endocervical adenocarcinoma, and this could lead to the misdiagnosis of the tubal involvement by an endocervical adenocarcinoma, such as STIC (36). In this study, we noted that both cases (cases 3 and 4) of gastrictype endocervical adenocarcinoma exhibited diffuse and strong nuclear p53 immunoreactivity (p53 over-expression) in both primary and tubal metastatic tumors. Correlation with the cervical findings and the mucinous features could result in an accurate diagnosis. p16 immunoreactivity is a reliable surrogate marker for HPV-associated endocervical adenocarcinoma, wherein the tumor cells show horizontally continuous, strong staining in almost all tumor cell nuclei. However, similar to p53 expression, this block positivity for p16 can be a misleading clue. Although the majority of STICs show generally negative or non-block p16 immunoreactivity (36), a small subset of STICs is diffusely and strongly positive for p16 regardless of high-risk HPV infection. In this situation, HPV in situ hybridization (ISH) is useful for recognizing the distribution of HPV within the tumor cells. HPV ISH is negative in STICs because HPV infection is irrelevant to STICs.

In addition, endometrioid tubal intraepithelial neoplasia (ETIN), also known as atypical endometrioid proliferation of the fallopian tube, should be included in the differential diagnosis of tubal intraepithelial lesions. In a recent study by 
Hecht et al. (35) who reported four cases of ETIN, the authors observed ETIN in $<1 \%$ of the hysterectomy specimens that were examined. ETIN is a focal and microscopic lesion that shows atypical glands with endometrioid cytomorphology that resembles endometrial atypical hyperplasia/endometrioid intraepithelial neoplasia. Similar to metastatic lesions, ETIN consists of cells with nuclear atypia, including hyperchromasia, pleomorphism, loss of polarity, and prominent nucleoli. In addition to these features, ETIN exhibits pale, oval nuclei and abundant cytoplasm with apical cytoplasmic vacuoles, which can mimic metastatic endocervical adenocarcinoma. Nuclear expression of $\beta$-catenin lends support to a diagnosis of ETIN rather than to a diagnosis of metastasis.

Intraepithelial spread of metastatic tumor cells has been described in other gynecological organs. The best recognized is vulvar intraepidermal metastases by colorectal and urothelial carcinomas, that is, secondary vulvar Paget disease (36). Metastatic tumors may involve the tubal surface epithelium by any of the following potential mechanisms: 1) extension of tumor deposits along the parametrium or serosal surface into the fimbrial mucosa (transperitoneal spread); 2) transuterine/transtubal spread of exfoliated tumor cells; 3 ) lymphovascular spread; and 4) direct extension. Two or more mechanisms may be involved in the tubal metastases. The transuterine/transtubal route has been suggested as a mode of spread of endocervical adenocarcinoma $(37,38)$. Of note, considering that all cases in this study extensively involved the endomyometrium, the most likely mechanism for the metastasis is that exfoliated tumor cells might spread along the uterine corpus into the isthmic portion of the tube. Furthermore, although the transperitoneal spread is suspected by the presence of parametrial extension in all cases, the metastatic tumors skipped the ovaries and peritoneum and involved the tubes only.

Data on the clinical significance of tubal IEM from cervical carcinomas are very limited, and we found no relevant literature on this issue. In our series, tubal IEMs were detected in cases with at least locally advanced disease, and all had pelvic lymph node metastases. One case had distant metastases at the time of surgery, and three of the remaining four cases developed metachronous distant metastases during the follow-up period. The mode of tumor spread within the gynecological organs is considered to affect the prognosis, as there are two contrasting biological forms of these: one in which a generally indolent tumor spreads transtubally, and another wherein aggressive disease spreads via conventional routes of dissemination (12). The spread of transuterine/transtubal metastases to the fallopian tube may not be an independent prognostic factor, but could represent an incidental finding that can be seen in locally advanced disease, especially in cases with endomyometrial involvement.
Neuroendocrine tumors are rare at all locations in the female genital tract (39). Tubal involvement of high-grade neuroendocrine carcinoma of gynecological origin has been mentioned in only a few reports in the literature $(39,40)$. In a recent case report, primary ovarian large cell neuroendocrine carcinoma (LCNEC) secondarily spread to the tubal mucosa (39). The pathological diagnosis of ovarian LCNEC in a 72year-old woman was rendered based on a typical neuroendocrine histology and positive immunoreactivities for neuroendocrine markers. The metastatic tumor involved the tubal mucosa with STIC-like and pagetoid growth patterns. To the best of our knowledge, our case is the first reported case of tubal IEM of primary cervical SCNEC. Based on the theory that the tubal intraepithelial carcinoma with expression of neuroendocrine markers and absence of WT1 suggests a precursor of primary neuroendocrine carcinoma of the fallopian tube (39), the possibility of intraepithelial involvement of primary tubal neuroendocrine tumors or a neuroendocrine component of another histological subtype exists. However, the advanced stage of disease at the time of diagnosis and the simultaneous presence of distant metastases support the possibility of a metastasis rather than that of a precursor of an independent primary tumor.

In summary, we described the clinicopathological characteristics of tubal IEMs of cervical carcinomas. Although ovarian metastasis was absent, all tubal metastatic tumors were detected microscopically and measured $\leq 2 \mathrm{~mm}$ in their greatest dimension. Tubal metastases from the cervical carcinoma should be distinguished from various tubal conditions including STIC, HGSC, and other nonneoplastic lesions. As the metastatic tumor resembles the primary tumor, morphological consistency between the primary and metastatic tumors can help to distinguish tubal metastasis from STIC. Moreover, immunostaining helps guide the differential diagnosis for challenging intraepithelial lesions of the fallopian tube. One of the possible mechanisms of tubal metastasis is transuterine/transtubal spread of exfoliated tumor cells. We believe that sharing the details of our clinical experience with tubal IEM will be of great help to further the pathologist's understanding of this rare condition and to establish an accurate diagnosis.

\section{Conflicts of Interest}

None of the Authors have any conflicts of interest or financial ties to declare regarding this study.

\section{Authors' Contributions}

All Authors made substantial contributions to the conception and design of the study; the acquisition, analysis, and interpretation of the data; drafting of the article; critical revision of the article for important intellectual content; and the final approval of the version to be published. 


\section{References}

1 Quinn M, Babb P, Jones J and Allen E: Effect of screening on incidence of and mortality from cancer of cervix in England: evaluation based on routinely collected statistics. BMJ 318(7188): 904-908, 1999. PMID: 10102852. DOI: 10.1136/bmj.318.7188.904

2 Arbyn M, Redman CWE, Verdoodt F, Kyrgiou M, Tzafetas M, Ghaem-Maghami S, Petry KU, Leeson S, Bergeron C, Nieminen $\mathrm{P}$, Gondry J, Reich $\mathrm{O}$ and Moss EL: Incomplete excision of cervical precancer as a predictor of treatment failure: a systematic review and meta-analysis. Lancet Oncol 18(12): 1665-1679, 2017. PMID: 29126708. DOI: 10.1016/S14702045(17)30700-3

3 Kalliala I, Athanasiou A, Veroniki AA, Salanti G, Efthimiou O, Raftis N, Bowden S, Paraskevaidi M, Aro K, Arbyn M, Bennett $\mathrm{P}$, Nieminen P, Paraskevaidis E and Kyrgiou M: Incidence and mortality from cervical cancer and other malignancies after treatment of cervical intraepithelial neoplasia: a systematic review and meta-analysis of the literature. Ann Oncol 31(2): 213-227, 2020. PMID: 31959338. DOI: 10.1016/j.annonc. 2019.11.004

4 Fontham ETH, Wolf AMD, Church TR, Etzioni R, Flowers CR, Herzig A, Guerra CE, Oeffinger KC, Shih YT, Walter LC, Kim JJ, Andrews KS, DeSantis CE, Fedewa SA, ManassaramBaptiste D, Saslow D, Wender RC and Smith RA: Cervical cancer screening for individuals at average risk: 2020 guideline update from the American Cancer Society. CA Cancer J Clin 70(5): 321-346, 2020. PMID: 32729638. DOI: 10.3322/ caac. 21628

5 Siegel RL, Miller KD and Jemal A: Cancer statistics, 2020. CA Cancer J Clin 70(1): 7-30, 2020. PMID: 31912902. DOI: $10.3322 /$ caac. 21590

6 Smith HO, Tiffany MF, Qualls CR and Key CR: The rising incidence of adenocarcinoma relative to squamous cell carcinoma of the uterine cervix in the United States - a 24-year population-based study. Gynecol Oncol 78(2): 97-105, 2000. PMID: 10926787. DOI: 10.1006/gyno.2000.5826

7 Tempfer CB, Tischoff I, Dogan A, Hilal Z, Schultheis B, Kern $P$ and Rezniczek GA: Neuroendocrine carcinoma of the cervix: a systematic review of the literature. BMC Cancer 18(1): 530, 2018. PMID: 29728073. DOI: 10.1186/s12885-018-4447-x

8 Zhou S and Peng F: Patterns of metastases in cervical cancer: a population-based study. Int J Clin Exp Pathol 13(7): 1615-1623, 2020. PMID: 32782680 .

9 Reyes C, Murali R and Park KJ: Secondary involvement of the adnexa and uterine corpus by carcinomas of the uterine cervix: a detailed morphologic description. Int J Gynecol Pathol 34(6): 551-563, 2015. PMID: 26166722. DOI: 10.1097/PGP 0000000000000206

10 Kim JY, Na K and Kim HS: Clinicopathological characteristics of mitotically-active cellular fibroma of the ovary: a singleinstitutional experience. Anticancer Res 37(5): 2557-2564, 2017. PMID: 28476827. DOI: 10.21873/anticanres.11599

11 Rabban JT, Vohra P and Zaloudek CJ: Nongynecologic metastases to fallopian tube mucosa: a potential mimic of tubal high-grade serous carcinoma and benign tubal mucinous metaplasia or nonmucinous hyperplasia. Am J Surg Pathol 39(1): 35-51, 2015. PMID: 25025442. DOI: 10.1097/PAS.0000000000 000293
12 Stewart CJ, Leung YC and Whitehouse A: Fallopian tube metastases of non-gynaecological origin: a series of 20 cases emphasizing patterns of involvement including intra-epithelial spread. Histopathology 60(6B): E106-E114, 2012. PMID: 22394169. DOI: 10.1111/j.1365-2559.2012.04194.x

$13 \mathrm{Na} \mathrm{K}$ and Kim HS: Clinicopathologic and molecular characteristics of mesonephric adenocarcinoma arising from the uterine body. Am J Surg Pathol 43(1): 12-25, 2019. PMID: 29189288. DOI: 10.1097/PAS.0000000000000991

14 Jung YY, Woo HY and Kim HS: Targeted genomic sequencing reveals novel TP53 in-frame deletion mutations leading to p53 overexpression in high-grade serous tubo-ovarian carcinoma. Anticancer Res 39(6): 2883-2889, 2019. PMID: 31177126. DOI: 10.21873/anticanres.13417

15 Jung H, Bae GE, Kim HM and Kim HS: Clinicopathological and molecular differences between gastric-type mucinous carcinoma and usual-type endocervical adenocarcinoma of the uterine cervix. Cancer Genomics Proteomics 17(5): 627-641, 2020. PMID: 32859641. DOI: 10.21873/cgp.20219

16 Park S, Cho EY, Oh YL, Park YH and Kim HS: Primary peritoneal high-grade serous carcinoma misinterpreted as metastatic breast carcinoma: a rare encounter in peritoneal fluid cytology. Anticancer Res 40(5): 2933-2939, 2020. PMID: 32366445. DOI: 10.21873/anticanres.14271

17 Park CK, Kim YW, Koh HH, Yoon N, Bae GE and Kim HS: Clinicopathological characteristics of squamous cell carcinoma and high-grade squamous intraepithelial lesions involving endocervical polyps. In Vivo 34(5): 2613-2621, 2020. PMID: 32871791. DOI: 10.21873/invivo.12079

18 Choi S, Joo JW, Do SI and Kim HS: Endometrium-limited metastasis of extragenital malignancies: a challenge in the diagnosis of endometrial curettage specimens. Diagnostics (Basel) 10(3): 150, 2020. PMID: 32164210. DOI: 10.3390/ diagnostics 10030150

19 Hwang S, Kim BG, Song SY and Kim HS: Ovarian gynandroblastoma with a juvenile granulosa cell tumor component in a postmenopausal woman. Diagnostics (Basel) 10(8): 537, 2020. PMID: 32751726. DOI: 10.3390/diagnostics 10080537

20 Köbel M, Ronnett BM, Singh N, Soslow RA, Gilks CB and McCluggage WG: Interpretation of P53 immunohistochemistry in endometrial carcinomas: toward increased reproducibility. Int J Gynecol Pathol 38(Suppl 1): S123-S131, 2019. PMID: 29517499. DOI: 10.1097/PGP.0000000000000488

$21 \mathrm{Na} \mathrm{K}$, Sung JY and Kim HS: TP53 mutation status of tuboovarian and peritoneal high-grade serous carcinoma with a wildtype p53 immunostaining pattern. Anticancer Res 37(12): 66976703, 2017. PMID: 29187446. DOI: 10.21873/anticanres.12128

22 Chung T, DO SI, Na K, Kim G, Jeong YI, Kim YW and Kim HS: Stromal p16 overexpression in gastric-type mucinous carcinoma of the uterine cervix. Anticancer Res 38(6): 35513558, 2018. PMID: 29848709. DOI: 10.21873/anticanres.12627

23 Darragh TM, Colgan TJ, Thomas Cox J, Heller DS, Henry MR, Luff RD, McCalmont T, Nayar R, Palefsky JM, Stoler MH, Wilkinson EJ, Zaino RJ, Wilbur DC and Members of the LAST Project Work Groups: The Lower Anogenital Squamous Terminology Standardization project for HPV-associated lesions: background and consensus recommendations from the College of American Pathologists and the American Society for Colposcopy and Cervical Pathology. Int J Gynecol Pathol 32(1): 
76-115, 2013. PMID: 23202792. DOI: 10.1097/PGP.0b013e $31826916 \mathrm{c} 7$

24 Sung JY, Jung YY and Kim HS: Clinicopathological characteristics and KRAS mutation status of endometrial mucinous metaplasia and carcinoma. Anticancer Res 38(5): 2779-2786, 2018. PMID: 29715099. DOI: 10.21873/anticanres. 12521

25 Park CK and Kim HS: Clinicopathological characteristics of ovarian metastasis from colorectal and pancreatobiliary carcinomas mimicking primary ovarian mucinous tumor Anticancer Res 38(9): 5465-5473, 2018. PMID: 30194204. DOI: 10.21873/anticanres.12879

$26 \mathrm{Na} \mathrm{K}$, Lee JY, Sung JY, Kim GM, Koo JS and Kim HS: Comparative clinicopathological and cytomorphological analyses of peritoneal carcinomatosis associated with metastatic breast carcinoma and primary peritoneal/ovarian carcinoma in patients with a history of breast carcinoma. Virchows Arch 473(2): 165175, 2018. PMID: 29926183. DOI: 10.1007/s00428-018-2390-5

$27 \mathrm{Na} \mathrm{K}$, Sung JY and Kim HS: Stromal p16 overexpression in adult granulosa cell tumors of the ovary. Anticancer Res 37(5): 2437-2444, 2017. PMID: 28476811. DOI: 10.21873/anticanres 11583

28 Kolin DL and Nucci MR: Fallopian tube neoplasia and mimics. Surg Pathol Clin 12(2): 457-479, 2019. PMID: 31097111. DOI: 10.1016/j.path.2019.01.006

29 Cass I, Holschneider C, Datta N, Barbuto D, Walts AE and Karlan BY: BRCA-mutation-associated fallopian tube carcinoma: a distinct clinical phenotype? Obstet Gynecol 106(6): 1327-1334, 2005. PMID: 16319259. DOI: 10.1097/ 01.AOG.0000187892.78392.3f

30 Hwang I, Lee J, Choi KH, Han J and Kim HS: Superficially spreading endocervical adenocarcinoma in situ with multifocal microscopic involvement of the endometrial surface: a case report with emphasis on the potential for misdiagnosis based on endometrial curettage specimens. Case Rep Oncol 13(3): 15301536, 2020. PMID: 33564294. DOI: 10.1159/000511568

31 WHO Classification of Tumors Editorial Board: WHO Classification of Tumours: Female Genital Tumours. IARC: Lyon, France, 2020.

32 Lee NK, Choi KU, Han GJ, Kwon BS, Song YJ, Suh DS and Kim KH: Pseudocarcinomatous hyperplasia of the fallopian tube mimicking tubal cancer: a radiological and pathological diagnostic challenge. J Ovarian Res 9(1): 79, 2016. PMID: 27842573. DOI: $10.1186 / \mathrm{s} 13048-016-0288-\mathrm{x}$
33 Cheung AN, Young RH and Scully RE: Pseudocarcinomatous hyperplasia of the fallopian tube associated with salpingitis. A report of 14 cases. Am J Surg Pathol 18(11): 1125-1130, 1994. PMID: 7943533. DOI: 10.1097/00000478-199411000-00006

34 Wong AK, Seidman JD, Barbuto DA, McPhaul LW and Silva EG: Mucinous metaplasia of the fallopian tube: a diagnostic pitfall mimicking metastasis. Int J Gynecol Pathol 30(1): 36-40, 2011. PMID: 21131836. DOI: 10.1097/PGP.0b013e3181f45f28

35 Hecht JL, Lin DI and Garrett L: Endometrioid tubal intraepithelial neoplasia (E-TIN) of the fallopian tube: A case series. Int J Gynecol Pathol 39(6): 552-557, 2020. PMID: 31855954. DOI: 10.1097/PGP.0000000000000650

36 Goldblum JR and Hart WR: Vulvar Paget's disease: a clinicopathologic and immunohistochemical study of 19 cases. Am J Surg Pathol 21(10): 1178-1187, 1997. PMID: 9331290. DOI: $10.1097 / 00000478-199710000-00008$

37 Chang MC, Nevadunsky NS, Viswanathan AN, Crum CP and Feltmate CM: Endocervical adenocarcinoma in situ with ovarian metastases: a unique variant with potential for long-term survival. Int J Gynecol Pathol 29(1): 88-92, 2010. PMID: 19952931. DOI: 10.1097/PGP.0b013e3181acefbf

38 Ronnett BM, Yemelyanova AV, Vang R, Gilks CB, Miller D, Gravitt PE and Kurman RJ: Endocervical adenocarcinomas with ovarian metastases: analysis of 29 cases with emphasis on minimally invasive cervical tumors and the ability of the metastases to simulate primary ovarian neoplasms. Am J Surg Pathol 32(12): 1835-1853, 2008. PMID: 18813124. DOI: 10.1097/PAS.0b013e3181758831

39 Vande Berg A, Segers K, Van de Vijver K, Van Rompuy AS and Colpaert C: Serous tubal intraepithelial carcinoma-like and pagetoid tubal metastasis of an ovarian large cell neuroendocrine carcinoma: peculiar metastatic growth patterns of a rare tumor. Int J Surg Pathol 29(3): 281-283, 2021. PMID: 33164612. DOI: $10.1177 / 1066896920972489$

40 Grondin K, Lidang M, Boenelycke M, Alvarado-Cabrero I, Herrington CS and McCluggage WG: Neuroendocrine tumors of the fallopian tube: report of a case series and review of the literature. Int J Gynecol Pathol 38(1): 78-84, 2019. PMID: 29019870. DOI: 10.1097/PGP.0000000000000458

Received April 19, 2021

Revised May 4, 2021

Accepted May 5, 2021 